Article

\title{
Stochastic Approaches to Deterministic Fluid Dynamics: A Selective Review
}

\author{
Ana Bela Cruzeiro \\ Departamento Matemática I.S.T. and Grupo de Física-Matemática University, Av. Rovisco Pais, \\ 1049-001 Lisbon, Portugal; ana.cruzeiro@tecnico.ulisboa.pt
}

Received: 20 January 2020; Accepted: 13 March 2020; Published: 19 March 2020

check for updates

\begin{abstract}
We present a stochastic Lagrangian view of fluid dynamics. The velocity solving the deterministic Navier-Stokes equation is regarded as a mean time derivative taken over stochastic Lagrangian paths and the equations of motion are critical points of an associated stochastic action functional involving the kinetic energy computed over random paths. Thus the deterministic Navier-Stokes equation is obtained via a variational principle. The pressure can be regarded as a Lagrange multiplier. The approach is based on Itô's stochastic calculus. Different related probabilistic methods to study the Navier-Stokes equation are discussed. We also consider Navier-Stokes equations perturbed by random terms, which we derive by means of a variational principle.
\end{abstract}

Keywords: Navier-Stokes equation; stochastic Lagrangian flows; stochastic variational principles; stochastic geometric mechanics

\section{Introduction}

The dynamics of an incompressible viscous fluid is modeled by the Navier-Stokes equation, a second order, nonlinear, partial differential equation describing the balance of mass and momentum of the fluid flow. In the absence of external forces and considering a perfectly incompressible fluid, the Navier-Stokes equation reads

$$
\frac{\partial}{\partial t} u+(u . \nabla) u=v \Delta u-\nabla p
$$

where the velocity field $u$ is required to satisfy the incompressibility condition $\operatorname{div} u=0$, the fluid density being equal to 1 . The constant $v$ denotes the kinematic viscosity and $u$ the fluid velocity. Moreover, the symbol $p$ stands for the pressure within the fluid, and is yet another unknown in the equation. Equation (1) has a huge number of applications in physics and engineering.

The Navier-Stokes equation is deterministic, but it is well known that some of its solutions seem to exhibit random behavior, which might eventually provide an insight into the onset of turbulence although from a purely mathematical point of view, the very important problem of existence and smoothness of the solutions to Equation (1) remains largely unsolved to this day. In this article we will review various ways of introducing randomness into the analysis of the solutions to Equation (1).

In Physics, the most famous deterministic equation hiding randomness is the Schrödinger equation. There is no mathematical probability theory behind it, but there is manifestly randomness in any Quantum Physics Lab. This puzzling situation did not prevent Quantum Theory to develop an impressive corpus of techniques to control, in particular, the transition from (in principle differentiable) solutions of classical equations of motion to some forms of randomness. Is it possible to draw an analogy between this classical/quantum relation and the one between Euler and Navier-Stokes equations? Of course the status of the two hydrodynamical equations are quite different from the 
above ones. Euler equation, although modeling only "dry water" (Von Neumann) is already quite complicated. It does not seem, yet, to have proof that it does not produce singularities. But the abovementioned analogy could, in particular, provide an insight into the onset of turbulence.

There are different ways to introduce randomness: uncertainty may have its origin in errors in the initial conditions, for example. In this case statistical approaches are considered: one studies the time evolution of some probability measure, supported on the relevant physical initial data. This is part of the statistical approach to turbulence, initiated already in the 19th century (c.f, among many others, [1,2]). Other various types of Langevin dynamics, using stochastic diffusion processes, have been proposed to describe equilibrium and non-equilibrium dynamics as well as Kraichnan's model in turbulent advection (c.f, for instance, [3]). On the other hand uncertainty may be generated by the chosen numerical model (we refer to [4] for a discussion on these issues in climate modeling, see also [5]).

Another very popular way to introduce stochasticity is to perturb the Navier-Stokes equation with random forces, so that stochastic partial differential equations then come into play. There is a huge literature in this subject, after the pioneering mathematical work [6]. Stochasticity is typically introduced at a Eulerian level, although some stochastic Lagrangian models of Langevin type (with smooth Lagrangian trajectories and stochastic velocities) have also been considered in turbulence ([7]).

More recently stochastic advection by Lie transport was introduced by D.D. Holm in [8]. The resulting equations of motion are stochastic partial differential equations and the approach is also Eulerian.

It would be impossible to mention here all stochastic approaches to fluid dynamics. We have only chosen some topics and a few corresponding references. It is worthwhile to mention that there are some interesting probabilistic representation formulae. Representing solutions of partial differential equations as expected values of functionals of stochastic processes is a tradition in the field of stochastic analysis and has also been considered for fluid dynamics. Very roughly speaking, we can find three different approaches: the probabilistic representation of the vorticity field as in [9], the analysis through branching processes and the Fourier transform as in [10] and that using Lagrangian diffusion processes as in [11].

On the other hand, in this paper, we are concerned with variational principles for deterministic dissipative fluid dynamics, and a brief state-of-the-art review follows. There are relatively few references on the special stochastic view of deterministic fluid dynamics advocated here. The oldest we know are [12,13], where the Laplacian term of the Navier-Stokes equation was interpreted, quite informally, as the presence of an underlying Brownian motion. In the famous paper [14], as well as in $[15,16]$, a rigorous geometric strategy for the Euler equation was developed. These geometric ideas have been extended by us to the Navier-Stokes equation, together with the associated variational principle, giving rise to a new stochastic geometric approach to dissipative dynamics. Recently, [17,18], more physical-oriented works, were influenced by references $[12,13]$ and also by our work.

The review paper presented here is, of course, very selective, not in terms of the qualities of quoted references, but in terms of their perspectives, in relation with the interplay between deterministic and stochastic viewpoints. We apologize to the many authors whose important works we were not able to describe here. We hope, however, to describe a special viewpoint in a consistent manner.

In a nutshell, the goal of this review is to show that probabilistic methods play a central role in the study of the deterministic Navier-Stokes equation, both from a conceptual and a practical point of view. Indeed, on the one hand the solutions to that equation satisfy stochastic variational principles. On the other hand, since the solutions to the Navier-Stokes equation may be interpreted as drifts of diffusion processes, one may import many techniques from stochastic analysis to investigate them. Those techniques include the use of stochastic differential equations, of forward-backward stochastic systems and of related numerical techniques. 


\section{Results}

As a dissipative system, there are well known obstructions if we want to derive the classic Navier-Stokes equation from a deterministic variational principle. Allowing the Lagrangian paths to be random and using Itô differential calculus (c.f [19]), we show how we can still derive the Navier-Stokes equation, without any randomness in the external forces, from a (stochastic) variational principle, where the Lagrangian functional is still the classic one, but computed over random Lagrangian trajectories. In some sense, the equation can be regarded as a generalized geodesic equation defined in some suitable space and the variational principle reduces to Hamilton's principle for the incompressible Euler equation when the viscosity vanishes. Our variational approach can be extended in order to consider stochastic Navier-Stokes equations as well.

After recalling Arnold's variational approach to the Euler equation, we describe in Section 4 two stochastic variational principles for the Navier-Stokes equation. The first is a "direct" generalization of Arnold's variational principle to the viscous case, the viscosity being associated with the random behavior of the particles. Incompressibility is incorporated in the definition of their trajectories. The second imposes incompressibility via a Lagrange multiplier.

Having justified our approach of Navier-Stokes equation using stochastic Lagrangian paths, Section 5 is devoted to several possible mathematical methods to study such paths. We can use the theory of forward-backward stochastic differential equations: this is explained in Section 5.1. We can also use entropy methods, since our action functional is essentially given by an entropy quantity. A notion of weak solutions, in the spirit of Brenier's work for the Euler equation and of optimal transport theory, allows us to consider cases where other methods are not accessible by lack of regularity. In Section 6 we describe some stability properties of the Navier-Stokes stochastic Lagrangian flows. The following paragraph is devoted to stochastic perturbations of the Navier-Stokes equation: we show that they can also be derived from a variational principle. Other equations and methods are mentioned in Section 8, as well as some future research problems. Finally a brief appendix contains basic notions of Itô stochastic calculus that are used in this paper.

\section{The Non Viscous Case}

As pointed out by Arnold ([14]), the incompressible Euler equation, corresponding to the case where the viscosity of the fluid is equal to zero, is an equation of geodesics on a suitable (infinite dimensional) space of functions. If we consider Lagrangian paths $g(t, x)$ such that $\frac{\partial}{\partial t} g(t, x)=$ $u(t, g(t, x))$, with $u$ solution of the Euler equation

$$
\frac{\partial}{\partial t} u+(u . \nabla) u=-\nabla p, \quad \operatorname{div} u=0,
$$

the acceleration $\frac{\partial^{2}}{\partial t} g$ is equal to a gradient function and thus, at every time, orthogonal (for the $L^{2}$ scalar product) to vector fields of zero divergence. This means that, if we endow the space of diffeomorphisms preserving the volume measure $m$ of the underlying configuration space a structure of manifold, the Lagrangian flows $g(t, \cdot)$ will be geodesics in such a manifold since its tangent space will consist of divergence free vector fields. In particular they will be critical points of the action functional defined by the kinetical energy:

$$
S[g]=\frac{1}{2} \int_{0}^{T} \int|\dot{g}(t, x)|^{2} d m(x) d t=\frac{1}{2} \int_{0}^{T}\|\dot{g}(t)\|_{L^{2}(d m)}^{2} d t .
$$

More precisely, Arnold showed that the Euler equation above corresponds to the equation of the geodesic flow of the (right-invariant) $L^{2}$ metric on the group of diffeomorphisms of the underlying configuration space that preserve the volume measure and have a certain Sobolev regularity. The velocity can be recovered from the Lagrangian flow: $u(t, x)=\left(\frac{\partial}{\partial t} g_{t}\right)\left(g_{t}^{-1}(x)\right)$. 
This program was rigorously developed in [15] and geodesics were shown to exist locally and under certain regularity restrictions on the initial conditions. Moreover the information on the geometry of the problem had important consequences, for instance in describing the chaotic behavior of Euler Equation (c.f [16]) and its consequences for weather prediction. Instability of the geodesic Euler flows can be described in terms of the sectional curvatures of the group of volume preserving diffeomorphisms. Explicit estimates for the curvatures, being non positive, show that, essentially, the weather is unpredictable.

Notice that this is a special case of Lagrangian system treated in Geometric Mechanics via variational principles on Lie groups ([20]). Indeed the space of volume preserving diffeomorphisms has also a group structure for the composition of maps and the $L^{2}$ metric is right-invariant.

\section{The Deterministic Navier-Stokes Equation}

The situation concerning variational principles for the Navier-Stokes equation is radically different since, contrary to the Euler one, this system is dissipative. Our approach describes dissipation through an introduction of noise in the Lagrangian trajectories. We replace deterministic Lagrangian paths by semimartingales of the form

$$
d \xi_{t}(x)=d M_{t}(x)+D_{t} \xi_{t}(x), \quad \xi_{0}(x)=x
$$

where $M_{t}$ is a martingale (for instance, a Brownian motion) and $D_{t} \xi_{t}$ denotes the bounded variation part of the semimartingale. The use of the notation $D_{t}$ is not an accident, since it corresponds to a mean derivative in time (recall that, almost-everywhere, $\xi_{t}$ satisfying Equation (3) will not be time-differentiable). Consider the definition of the generalized derivative $D_{t}$. Namely, for every regular function $F$,

$$
D_{t} F\left(t, \xi_{t}\right)=\lim _{\epsilon \rightarrow 0} \frac{1}{\epsilon} E_{t}\left[F\left(t+\epsilon, \xi_{t+\epsilon}\right)-F\left(t, \xi_{t}\right)\right],
$$

where $E_{t}$ denotes the conditional expectation with respect to the past information of the process $\xi$. Applying this definition to the identity function, and since the operator $D_{t}$ vanishes when considered on martingales, we see that $D_{t} \xi_{t}$ coincides with the drift of the process and can indeed be understood as a regularized derivative. This notion has been known in Stochastic Analysis since its beginnings, as it corresponds to the definition of the generator of the process $\xi_{t}$, but it became more relevant in dynamics with the works of Nelson [21].

When $\xi$ takes values in a non-Euclidean space, notably a Riemannian manifold or a Lie group, one can extend the definition of generalized derivative using parallel transport. In this paper we will mainly consider the Euclidean setting. We denote the configuration space by $\mathcal{O}$ and, for now, we assume that $\mathcal{O}$ has no boundary (case with periodic boundary conditions, for example).

\subsection{Stochastic Geometric Mechanics Approach}

We consider the space $G_{V}$ of bijective maps $g: \mathcal{O} \rightarrow \mathcal{O}$ which belong to $L^{2}=L^{2}(d m)$ and keep the volume measure invariant, namely such that $\int f(g(x)) d m(x)=\int f d m(x)$ for every (continuous) function $f$. This infinite dimensional space of maps has a group structure under composition and a Riemannian one with respect to the $L^{2}$ metric ([15]). The tangent space to the identity map consists of vector fields on $\mathcal{O}$ with zero divergence. Moreover a simple covariant derivative can be defined: for such two vector fields $X$ and $Y, \nabla_{X} Y=\Pi\left(\partial_{X} Y\right)$ where $\partial$ is the usual Lie derivative and $\Pi$ the projection operator from $L^{2}$ to divergence free vectors associated to the Helmholtz (or Hodge, according to the source) decomposition.

The next result was proved in [22], then extended to Riemannian manifolds in [23] and formulated on general Lie groups in [24]. 
We consider a stochastic action functional where the Lagrangian is the kinetic energy computed on the generalized derivative of a semimartingale. The corresponding norm is the $L^{2}$ one. More precisely, for a $G_{V}$-valued semimartingale $\xi$ as in Equation (4) we define

$$
S[\xi]=\frac{1}{2} E \int_{0}^{T} \int_{\mathcal{O}}\left|D_{t} \xi_{t}(x)\right|^{2} d t d m(x)
$$

where $E$ means expectation with respect to the underlying probability. A particular class of such semimartingales is the following. To a time dependent vector field with zero divergence $u(t, \cdot)$, $t \in[0, T]$, and belonging to $L^{2}$, we associate the stochastic differential equation (c.f [19], for example, as a reference for Itô stochastic calculus, as well as the Appendix A in this article),

$$
d g_{t}^{u}(x)=\sqrt{2 v} d W_{t}+u\left(t, g_{t}^{u}(x)\right) d t, \quad g_{0}^{u}(x)=x
$$

where $W$ is a standard Brownian motion. This equation defines a stochastic flow of maps on $\mathcal{O}$ that belong to $G_{V}$ and satisfy $D_{t} g_{t}^{u}=u\left(t, g_{t}\right)$.

Considering a simple Brownian motion may be regarded as oversimplifying. Actually one should introduce martingales driven by vector fields modeling the correlations observed in the physical model ([8]). Typically Lagrangian paths are of the form

$$
d g_{t}=\sum_{k} H_{k}\left(g_{t}\right) d W_{t}^{k}+u\left(t, g_{t}\right) d t
$$

where $H_{k}$ are correlation eigenvectors and $W^{k}$ independent Brownian motions. Our approach covers such cases $([22,24,25])$ and we chose here a Brownian motion only to simplify the exposition.

We are interested in derivating $S\left[g^{u}\right]$; in particular we want to consider variations of the paths $g^{u}$ for which the functional above is still well defined, i.e., they are still $G_{V}$-valued semimartingales. Consider the exponential type functions

$$
e_{t}(\epsilon v)(x)=x+\epsilon \int_{0}^{t} \dot{v}\left(s, e_{s}(\epsilon v)(x)\right) d s
$$

with $\epsilon>0$ and where $v(t, \cdot)$ is a smooth time dependent vector field such that $v(0)=v(T)=0$ and $\operatorname{div} v(t, \cdot)=0$ for every $t \in[0, T]$. Notice that, up to the first order in $\epsilon$, we have $e_{t}(\epsilon v)(x) \simeq$ $x+\epsilon v(t, x)$. The variations of the paths $g^{u}(t)$ will be defined by left composition, since the functional is right-invariant:

$$
g_{t}^{u, \epsilon}=e_{t}(\epsilon v) \circ g^{u}(t)
$$

We have, using Itô calculus,

$$
\left.d g_{t}^{u, \epsilon}=\nabla e_{t}(\epsilon v)\left(g_{t}^{u}\right) \sqrt{2 v} d W_{t}+\left[\dot{e}_{t}(\epsilon v)+(u . \nabla) e_{t}(\epsilon v)+v \Delta e_{t}(\epsilon v)\right]\left(g_{t}^{u}\right)\right] d t
$$

By the definition of $e_{t}(v)$,

$$
\left.\frac{d}{d \epsilon}\right|_{\epsilon=0} S\left[e .(\epsilon v) \circ g^{u}(\cdot)\right]=E \int_{0}^{T}\left(\int D_{t} g^{u}(t)(x) \cdot D_{t} v\left(g^{u}(t)(x)\right) d m(x)\right) d t
$$

and by Itô's formula,

$$
\begin{aligned}
& d \int D_{t} g^{u}(t)(x) \cdot v\left(g^{u}(t)(x)\right) d m(x)=\int d D g^{u}(t)(x) \cdot v\left(g^{u} u(t)(x)\right) d m(x)+\int D g^{u}(t)(x) \cdot d v\left(g^{u}(t)(x)\right) d m(x) \\
&+\int d D_{t} g^{u}(t)(x) \cdot d v\left(g^{u}(t)(x)\right) d m(x)
\end{aligned}
$$


The last (Itô's contraction) term is equal to

$$
2 v\left(\int(\nabla v \otimes \nabla u)\left(g^{u}(t)(x)\right) d m(x)\right) d t
$$

As $v(0)=v(T)=0$ this implies,

$$
\left.\frac{d}{d \epsilon}\right|_{\epsilon=0} S\left[e .(\epsilon v) \circ g^{u}(\cdot)\right]=-E \int_{0}^{T}\left(\int\left(D_{t} D_{t} g^{u}(t)(x) d m(x)\right) d t-2 v E \int_{0}^{T}\left(\int(\nabla v \otimes \nabla u)\left(g^{u}(t)(x)\right) d m(x)\right) d t\right.
$$

On the other hand

$$
D_{t} D_{t} g^{u}(t)=D_{t} u\left(t, g^{u}(t)\right)=\left(\frac{\partial}{\partial t} u+(u . \nabla) u+v \Delta u\right)\left(g^{u}(t)\right) .
$$

Therefore, using the invariance of the measure with respect to the process $g^{u}$ and integration by parts, we obtain

$$
\begin{gathered}
\left.\frac{d}{d \epsilon}\right|_{\epsilon=0} S\left[e .(\epsilon v) \circ g^{u}(\cdot)\right]=-E \int_{0}^{T} \int\left(\left(\left[\frac{\partial}{\partial t} u+(u . \nabla) u-v \Delta u\right] \cdot v\right)\left(t, g^{u}(t)(x)\right) d m(x)\right) d t \\
=-\int_{0}^{T}\left(\int\left(\left[\frac{\partial}{\partial t} u+(u . \nabla) u-v \Delta u\right] \cdot v\right)(t, x) d m(x)\right) d t
\end{gathered}
$$

for every $v$ with zero divergence, which means that $\frac{\partial}{\partial t} u+(u \cdot \nabla) u-v \Delta u$ is the gradient of some function.

We have therefore the following

Theorem 1. Let $u(t, \cdot)$ be a smooth time dependent divergence-free vector field defined on $[0, T] \times \mathcal{O}$. Let $g^{u}(t)$ be a stochastic Brownian flow with diffusion coefficient $\sqrt{2 v}$ and drift $u$ (as in Equation (7)). Then $g^{u}$ is a critical point of the energy functional $S$ if and only if there exists a function $p$ such that $u(t)$ verifies the incompressible Navier-Stokes Equation (1).

Remark 1. When $\mathcal{O}$ is a Riemannian manifold (say, without boundary), we can still define an action functional of the form in Equation (6), but some more concepts are needed.

In that case the Itô differential of an $\mathcal{O}$-valued semimartingale $Y$ is defined by

$$
d Y_{t}=P(Y)_{t} d\left(\int_{0}^{\cdot} P(Y)_{s}^{-1} \circ d Y_{s}\right)_{t}
$$

where $P(Y)_{t}: T_{Y_{0}} M \rightarrow T_{Y_{t}} M$ is the parallel transport associated with the Levi-Civita connection along $t \mapsto Y_{t}$. Alternatively, in local coordinates,

$$
d Y_{t}=\left(d Y_{t}^{i}+\frac{1}{2} \Gamma_{j k}^{i}\left(Y_{t}\right) d Y_{t}^{j} \otimes d Y_{t}^{k}\right) \partial_{i}
$$

where $\Gamma_{j k}^{i}$ are the Christoffel symbols of this connection.

If the semimartingale $Y_{t}$ has an absolutely continuous drift, we denote it by $D Y_{t}$ : for every 1-form $\alpha \in \Gamma\left(T^{*} \mathcal{O}\right)$, the finite variation part of $\int_{0}^{\cdot}\left\langle\alpha\left(Y_{t}\right), d Y_{t}\right\rangle$ is $\int_{0}^{\cdot}\left\langle\alpha\left(Y_{t}\right), D Y_{t} d t\right\rangle$. We consider an incompressible Brownian flow $g^{u}(t)$ with covariance $a \in \Gamma(T \mathcal{O} \otimes T \mathcal{O})$ and time dependent drift $u(t, \cdot) \in \Gamma(T \mathcal{O})$. We assume that for all $x \in \mathcal{O}, a(x, x)=2 v \mathbf{g}^{-1}(x)$ for some $v>0$, where $\mathbf{g}$ denotes the metric tensor of the manifold. Such incompressible flows are known to be well defined on compact symmetric spaces and on compact Lie groups. 
This means that

$$
\begin{gathered}
d g_{u}(t)(x) \otimes d g_{u}(t)(y)=a\left(g_{u}(t)(x), g_{u}(t)(y)\right) d t, \\
d g_{u}(t)(x) \otimes d g_{u}(t)(x)=2 v \mathbf{g}^{-1}\left(g_{u}(t)(x)\right) d t,
\end{gathered}
$$

the drift of $g^{u}(t)(x)$ is absolutely continuous and satisfies $D g^{u}(t)(x)=u\left(t, g_{u}(t)(x)\right)$. Then, using the same kind of variations as in the flat case, we derive (c.f [23]), from the energy functional

$$
S\left(g^{u}\right)=\frac{1}{2} E\left[\int_{0}^{T}\left(\int_{\mathcal{O}}\left|D g_{u}(t)(x)\right|^{2} d m(x)\right) d t\right],
$$

the equation

$$
\frac{\partial}{\partial t} u+\nabla_{u} u=v L u-\nabla p
$$

where $L=d d^{*}+d^{*} d$ is the the Laplace-de Rham operator. We recall that when computed on forms and, in particular, on vector fields, $L$ differs from the usual Levi-Civita Laplacian by a Ricci curvature term.

\subsection{Lagrange Multipliers Approach}

Here we describe another stochastic variational principle for the Navier-Stokes equation, that uses a Lagrange multiplier formulation. Its advantage is that it does not need to be formulated in the space $G_{V}$, since incompressibility is not incorporated in the definition of the flows, but given instead by the multiplier condition. The problem becomes strictly finite-dimensional and variations of the paths may be defined simply by shifts. It also allows us to consider domains with boundary. References for the Lagrange multipliers approach are $[26,27]$, where one can find detailed proofs. The first concerns the case of a domain without boundary (more precisely the torus) and in the second one can find its extension to a domain with boundary.

Let $\mathcal{O}$ be a domain with a regular boundary. We consider the Navier-Stokes equation with Neumann boundary condition on $[0, T] \times \mathcal{O}$, namely the condition $\nabla u . n=0$, where $n$ denotes the unit vector normal to the boundary. The stochastic Lagrangian flows can now take into account reflections at the boundary of the domain and can be written,

$$
d g_{t}(x)=\sqrt{2 v} d W_{t}+u\left(t, g_{t}(x)\right) d t+n\left(g_{t}(x)\right) d \ell(t), \quad g_{0}(x)=x \in \overline{\mathcal{O}}
$$

where $\ell(t)=\int_{0}^{t} 1_{\delta \mathcal{O}}\left(g_{s}(x)\right) d \ell(s)$ is the local time, representing the amount of time spent by the diffusion process in the neighborhood of points in the boundary.

The action functional is defined as

$$
\begin{aligned}
S(g, p) & =\frac{1}{2} E \int_{0}^{T} \int\left|D_{t} g_{t}(x)\right|^{2} d t d m(x)+E \int_{0}^{T} \int p\left(t, g_{t}(x)\right)\left(\operatorname{det} \nabla g_{t}(x)-1\right) d t d m(x) \\
& :=S^{1}(g, p)+S^{2}(g, p)
\end{aligned}
$$

The extra term $S^{2}$ corresponds to a Lagrange multiplier whose constraint forces the paths to keep the volume measure preserved during the evolution (incompressibility condition). The variable $p$ is defined in the linear space $L^{2}([0, T] \times \mathcal{O})$. We consider variations of the form

$$
\begin{gathered}
g_{t}(\cdot) \rightarrow g_{t}^{\epsilon}(\cdot)=g_{t}(\cdot)+\epsilon h\left(t, g_{t}(\cdot)\right) \\
p(t, \cdot) \rightarrow p^{\epsilon}(t, \cdot)=p(t, \cdot)+\epsilon \varphi\left(t, g_{t}(\cdot)\right)
\end{gathered}
$$

with $h(t, x)$ and $\varphi(t, x)$ deterministic and smooth, satisfying $h(T, \cdot)=h(0, \cdot)=0, h=0$ on $\partial \mathcal{O}$. 
Concerning $S^{2}$, we have,

$$
\begin{aligned}
\left.\frac{d}{d \varepsilon}\right|_{\varepsilon=0} S^{2}\left(g^{\epsilon}, p^{\epsilon}\right) & =E \int_{0}^{T} \int \varphi\left(t, g_{t}(x)\right)\left(\operatorname{det} \nabla g_{t}(x)-1\right) d t d m(x) \\
& +E \int_{0}^{T} \int\left(\nabla p\left(t, g_{t}(x)\right) \cdot h\left(t, g_{t}(x)\right)\left(\operatorname{det} \nabla g_{t}(x)-1\right) d t d m(x)\right. \\
& +\left.E \int_{0}^{T} \int p\left(t, g_{t}(x)\right) \frac{d}{d \varepsilon}\right|_{\varepsilon=0} \operatorname{det} \nabla\left(g_{t}(x)+\epsilon h\left(t, g_{t}(x)\right) d t d m(x)\right.
\end{aligned}
$$

Since $\varphi$ is arbitrary we conclude from the first term of Equation (11) that critical points of the action are volume-preserving diffeomorphisms $\left(\operatorname{det} \nabla g_{t}(x)=1\right)$ and therefore have divergence-free drifts. It follows immediately that Equation (12) is also equal to zero. The computation of the third term gives

$$
\left.\frac{d}{d \varepsilon}\right|_{\varepsilon=0} S^{2}\left(g^{\epsilon}, p^{\epsilon}\right)=-E \int_{0}^{T} \int\left(\nabla p\left(t, g_{t}(x)\right) . h\left(t, g_{t}(x)\right)\right) d t d m(x) .
$$

Actually this last computation does not use stochastic calculus:

$$
\begin{gathered}
\left.\frac{d}{d \varepsilon}\right|_{\varepsilon=0} \operatorname{det} \nabla\left(g_{t}(x)+\epsilon h\left(t, g_{t}(x)\right)\right)=\operatorname{det} \nabla g_{t}(x) \operatorname{tr}\left(\left.\left(\nabla g_{t}(x)\right)^{-1} \frac{d}{d \varepsilon}\right|_{\varepsilon=0} \nabla g_{t}^{\epsilon}(x)\right) \\
=\operatorname{det}\left(\nabla g_{t}(x)\right) \operatorname{tr}\left(\left(\nabla g_{t}(x)\right)^{-1} \nabla\left(h\left(t, g_{t}(x)\right)\right)\right) .
\end{gathered}
$$

Since

$$
\begin{gathered}
\partial_{i}\left(p\left(t, g_{t}\right)\left(\nabla g_{t}\right)_{i j}^{-1} h\left(t, g_{t}\right)^{j}\right)=\partial_{i}\left(p\left(t, g_{t}\right)\right)\left(\nabla g_{t}\right)_{i j}^{-1} h\left(t, g_{t}\right)^{j}+p\left(t, g_{t}\right)\left(\nabla g_{t}\right)_{i j}^{-1} \partial_{i}\left(h^{j}\left(t, g_{t}\right)\right) \\
+p\left(t, g_{t}\right) h^{j}\left(t, g_{t}\right) \partial_{i}\left(\nabla g_{t}\right)_{i j}^{-1}, \\
(13)=-E \int_{0}^{T} \int\left[\partial_{i}\left(p\left(t, g_{t}\right)\right)\left(\nabla g_{t}\right)_{i j}^{-1}+p\left(t, g_{t}\right) \partial_{i}\left(\left(\nabla g_{t}\right)_{i j}^{-1}\right)\right] h^{j}\left(t, g_{t}\right) \operatorname{det} \nabla g_{t} d t d x .
\end{gathered}
$$

Notice that we already concluded that $\operatorname{det} \nabla g_{t}=1$. On the other hand,

$$
\sum_{i} \partial_{i}\left(\nabla g_{t}\right)_{i j}^{-1}=0
$$

Indeed, derivating the equality det $\nabla g_{t}=1$, we get

$$
\partial_{k} \operatorname{det}\left(\nabla g_{t}\right)=\operatorname{tr}\left(\left(\nabla g_{t}\right)^{-1} \partial_{k}\left(\nabla g_{t}\right)\right)=\sum_{i}\left(\nabla g_{t}\right)_{i j}^{-1} \partial_{k} \partial_{i} g_{t}^{j}=0 .
$$

Also, derivating equality $\left(\nabla g_{t}\right)_{i j}^{-1} \partial_{k} g_{t}^{j}=\delta_{i k}$, we obtain

$$
\sum_{i} \partial_{i}\left(\nabla g_{t}\right)_{i j}^{-1} \partial_{k} g_{t}^{j}+\left(\nabla g_{t}\right)_{i j}^{-1} \partial_{i} \partial_{k} g_{t}^{j}=0 ;
$$

therefore

$$
\sum_{i} \partial_{i}\left(\nabla g_{t}\right)_{i k}^{-1}=-\left(\left(\nabla g_{t}\right)_{i j}^{-1} \partial_{k} \partial_{i} g_{t}^{j}\right)\left(\nabla g_{t}\right)_{j k}^{-1}=0
$$

and

$$
\left.(13)=-E \int_{0}^{T} \int\left(\partial_{i}\left(p\left(t, g_{t}(x)\right)\right)\left(\nabla g_{t}(x)\right)_{i j}^{-1}\right) h_{t}^{j}\left(g_{t}(x)\right) \operatorname{det} \nabla g_{t}(x)\right) d t d m(x)
$$




$$
=-E \int_{0}^{T} \int\left(\nabla p\left(t, g_{t}(x)\right) \cdot h\left(t, g_{t}(x)\right)\right) d t d m(x) .
$$

We now look at the derivation of $S^{1}$. We can prove, using Itô calculus and similarly to the computation in Section 4.1, that

$$
\begin{gathered}
\left.\frac{d}{d \varepsilon}\right|_{\varepsilon=0} S^{1}\left(g^{\epsilon}, p^{\epsilon}\right)=E<D g_{T}, h\left(T, g_{T}\right)>-E<D g_{0}, h\left(0, g_{0}\right)>-E \int_{0}^{T} \int\left(D_{t} D_{t} g_{t}(x) \cdot h\left(t, g_{t}(x)\right)\right) d t d x \\
-E \int_{0}^{T} \int\left(d D_{t} g_{t}(x) \cdot d h\left(t, g_{t}(x)\right)\right) d x \\
=-E \int_{0}^{T} \int\left(D_{t} D_{t} g_{t}(x) \cdot h\left(t, g_{t}(x)\right)\right) d t d m(x)-2 v E \int_{0}^{T}(\nabla u \cdot \nabla h)\left(t, g_{t}(x)\right) d t d m(x)
\end{gathered}
$$

Using equality $D_{t} D_{t} g_{t}(x)=\left(\frac{\partial}{\partial t} u+(u . \nabla) u+v \Delta u+n \nabla u \ell(t)\right)\left(t, g_{t}(x)\right)$ and integration by parts, we deduce that

$$
\left.\frac{d}{d \varepsilon}\right|_{\varepsilon=0} S^{1}\left(g^{\epsilon}, p^{\epsilon}\right)=-E \int_{0}^{T} \int\left[\left(\left(\frac{\partial}{\partial t} u+(u . \nabla) u-v \Delta u\right) . h\right)+(n . \nabla u) h \ell(t)\right]\left(t, g_{t}(x)\right) d t d m(x) .
$$

Combining the expressions above for the variation of the action functional and using the invariance of the volume measure for the flows, we obtain the following result,

Theorem 2. A diffusion $g_{t}$ of the form of Equation (8) and a function $p$ are critical for the action functional in Equation (9) if and only if the drift $u(t, \cdot)$ of $g_{t}$ satisfies the Navier-Stokes equation

$$
\partial_{t} u+(u . \nabla) u=v \Delta u-\nabla p, \quad \operatorname{div} u(t, \cdot)=0, \quad \nabla u . n=0 \text { in } \partial \mathcal{O}
$$

with $t \in[0, T]$.

\section{Constructing Solutions of Navier-Stokes Equation by Probabilistic Methods}

\subsection{Forward-Backward Stochastic Differential Systems}

The stochastic Lagrangian flows $g_{t}(\cdot)$ obtained either via the geometric or the Lagrangian multiplier approach described above satisfy an equation of Newton type. More precisely, on a fixed time interval $[0, T]$ and after the change of time $v(t, x)=-u(T-t, x)$, we have $D g_{t}(x)=$ $-v\left(T-t, g_{t}(x)\right)$ and

$$
D_{t} v\left(t, g_{t}(x)\right)=-\nabla p\left(t, g_{t}(x)\right), \quad g_{0}(x)=x
$$

or, regarded as an equation in the weak $L^{2}$ sense,

$$
D_{t} v\left(t, g_{t}(\cdot)\right)=0, \quad g_{0}=\mathrm{id}
$$

Formally, when the viscosity is zero, this is Arnold's geodesic equation and ours can be seen, indeed, as a generalized geodesic equation. Moreover, although we only consider in this paper an Euclidean setting, the framework can be extended without essential difficulties to a general Riemannian manifold or a Lie group $([23,24])$.

How does one solve directly this equation of motion? One possible way is to characterize it in terms of forward-backward stochastic differential systems, which are second order stochastic equations (c.f the Appendix A). We have formulated the problem in [28] first, then solved it in [29,30] for some specific function spaces and in two and three dimensions, respectively. The characterization in terms of forward-backward stochastic differential equations has also the advantage that it may 
allow to implement numerical methods, known for such systems (c.f for example, [31] and the recent work [32]).

The forward-backward system solved by our stochastic Lagrangian flows can be written in the form

$$
\left\{\begin{array}{c}
d g_{t}=\sqrt{2 v} d W_{t}+Y_{t} d t \\
d Y_{t}=Z_{t} d W_{t}-\nabla p\left(g_{t}\right) d t
\end{array}\right.
$$

together with a given initial condition for the forward equation $\left(g_{0}=x\right)$ and a final condition for the backward one, $Y_{T}$. It can be proved these type of systems are well posed and their solutions are of the form $Y_{t}=Y_{t}(x)=v\left(t, g_{t}(x)\right)\left(=-u\left(t, g_{t}(x)\right)\right)$ for some vector field $v$. We have,

$$
D_{t} Y_{t}=D_{t} D_{t} g_{t}=-\nabla p\left(g_{t}\right)
$$

(compare with Equation (15)). Variable $Z$, although a priori unknown, is a posteriori determined and equal to $\sqrt{2 v} \nabla v\left(g_{t}\right)$.

To be more precise, let $u$ be a solution of the Navier-Stokes equation in the time interval $t \in[0, T]$ and assume that $u$ is regular. Let $g_{s}^{t}(x)$ be the unique solution of the following stochastic differential equation,

$$
\left\{\begin{array}{l}
d g_{s}^{t}(x)=\sqrt{2 v} d W_{s}-u\left(T-s, g_{s}^{t}(x)\right) d s \\
g_{t}^{t}(x)=x,
\end{array}\right.
$$

with $s>t$. We define $Y_{s}^{t}(x)=u\left(T-s, g_{s}^{t}(x)\right), Z_{s}^{t}(x)=\nabla u\left(T-s, g_{s}^{t}(x)\right)$; applying Itô's formula directly, the following forward-backward stochastic differential system with solution $\left(g_{s}^{t}(x), Y_{s}^{t}(x), Z_{s}^{t}(x), u(t, x), p(t, x)\right)$ is derived,

$$
\left\{\begin{array}{l}
d g_{s}^{t}(x)=\sqrt{2 v} d W_{s}-u\left(T-s, g_{s}^{t}(x)\right) d s \\
d Y_{s}^{t}(x)=\sqrt{2 v} Z_{s}^{t}(x) d W_{s}-\nabla p\left(T-s, g_{s}^{t}(x)\right) d s \\
Y_{t}^{t}(x)=u(T-t, x) \\
g_{t}^{t}(x)=x, Y_{T}^{t}(x)=u_{0}\left(g_{T}^{t}(x)\right)
\end{array}\right.
$$

Recall also that the pressure satisfies the following identity

$$
\Delta p(t, x)=\sum_{i, j=1}^{3} \partial_{i} u^{j}(t, x) \partial_{j} u^{i}(t, x)
$$

On the other hand, if $\left(g_{s}^{t}(x), Y_{s}^{t}(x), Z_{s}^{t}(x), u(t, x), p(t, x)\right)$ is a solution of Equation (17) together with Equation (18) and $u$ is regular enough, then the vector field $u(t, x):=Y_{T-t}^{T-t}(x)$ satisfies the Navier-Stokes equation for $t \in[0, T]$. In particular, we can show that $\operatorname{div} u(t, x)=0$ due to the expression of $p(t, x)$ given by Equation (18).

In order to incorporate Equation (18) in the forward-backward system and obtain a closed system of equations we proceed as follows. Denote by $N$ the Newton's potential in $\mathbb{R}^{d}, d \geq 3$, i.e., the operator $\Delta^{-1}$ which is given by

$$
N f(x)=C(d) \int_{\mathbb{R}^{d}} \frac{f(y)}{|x-y|^{d-2}} d y,
$$

where $C(d)$ is a constant depending on the dimension $d$. Then we can write Equations (17) and (18) as

$$
\left\{\begin{array}{l}
d g_{s}^{t}(x)=\sqrt{2 v} d W_{s}-u\left(T-s, g_{s}^{t}(x)\right) d s \\
d Y_{s}^{t}(x)=\sqrt{2 v} Z_{s}^{t}(x) d W_{s}-\nabla N\left(\sum_{i, j} \partial_{i} v^{j}-\partial_{j} v^{i}\right)\left(T-s, g_{s}^{t}(x)\right) d s \\
Y_{t}^{t}(x)=u(T-t, x) \\
g_{t}^{t}(x)=x, Y_{T}^{t}(x)=u_{0}\left(g_{T}^{t}(x)\right),
\end{array}\right.
$$


Using suitable $L^{p}$ bounds of the operator $\nabla N\left(\sum_{i, j} \partial_{i} v^{j}-\partial_{j} v^{i}\right)$ we have constructed in [30] local unique solutions of the system in Equation (19), and therefore of the Navier-Stokes equation, in some Sobolev-type functional spaces in dimension $d \geq 3$. The two-dimensional case was studied in a similar way [29], but via the vorticity equation.

Such type of forward-backward differential equations were also studied on general Lie groups in [33].

\subsection{Entropy Methods}

In a closely related recent work ([34]), we explored the fact that the stochastic kinetic energy used here coincides in fact with a relative entropy, of the law of the diffusion process associated with Navier-Stokes in relation to the Wiener law. Brenier's relaxation of Arnold's geodesic problem, extended to the stochastic setting, becomes, in this sense, an entropy minimization problem.

Recall that the relative entropy of a probability measure $Q$ on some measurable set $X$ with respect to a reference probability measure $R$ on $X$ is defined as

$$
H(Q \mid R)=\int_{X} \log \left(\frac{d Q}{d R}\right) d Q
$$

when $Q$ is absolutely continuous with respect to $R$. What we have named the Brenier-Schrödinger problem in [34] consists of the following. Consider $X$ to be an Euclidean space and fix as reference measure $R$ as the law of the reversible Brownian motion on a time interval $[0, T]$, namely $R=$ $\int_{X} P^{x} d m(x)$ for $P^{x}$ the law of a Brownian motion starting at $x$. The we look for a measure $Q$ minimizing the relative entropy $H(Q \mid R)$ under the constraint that $Q_{t}$, the marginals at time $t$, are given, and that the joint law at initial and final times, $Q_{0 T}$, is also given. In our case the relevant condition for the marginals is the incompressibility condition $Q_{t}=m$ for all times.

This is a convex minimization problem reminiscent of the Schrödinger problem in Quantum Mechanics ([35-37]) and, simultaneously, a generalization of a Monge-Kantarovich mass transportation problem (c.f [38]).

The relation between the entropy problem and our stochastic variational one relies on the fact that, by Girsanov's theorem (c.f for instance [19]), if the Lagrangian path is given by the stochastic differential equation $d g_{t}^{u}=\sqrt{2 v} d W_{t}+u\left(t, g_{t}^{u}\right) d t$ with initial condition $R$, and if $Q$ denotes the law of this stochastic process, we have

$$
H(Q \mid R)=H\left(Q_{0} \mid R\right)+\frac{1}{2} E \int_{0}^{T}\left|u\left(t, g_{t}^{u}\right)\right|^{2} d t
$$

Therefore the entropy minimization problem corresponds to our stochastic variational principle, described here in Section 4. We can use then tools of convex analysis, establish the existence of a solution, consider a dual problem that provides the pressure term and exhibit Lagrange multipliers. Since our solution is the law of a stochastic process which is absolutely continuous with respect to the reference measure, one can write its Radon-Nikodym derivative and compare it to an alternative expression obtained from convex duality. We find that the drifts of our Lagrangian flows, written as velocity fields, have unusual properties: when stratified (conditioned to the arrival point of the stochastic process), the backward velocity is the gradient of the solution to a Hamilton-Jacobi-Bellman equation, while the pressure remains independent of the arrival point. We recover, with this method, a structure that is analogous to Brenier's stratified solutions in [39].

\subsection{A Weak Notion of Navier-Stokes Solutions}

One cannot expect that the stochastic variational approach will determine solutions of the Navier-Stokes in full generality. It is already the case that geodesics associated with Euler equation do not exist in some situations. The main difficulty is that the topology induced by the energy is not strong enough to deal with the needed regularity of the Lagrangian maps. In order to overcome the 
problem, Brenier has introduced a concept that he named "generalized solutions", replacing the notion of geodesic path by a probability measure over geodesic paths, in the spirit of the Monge-Kantarovich problem ([40]). An extension of Brenier's framework to the stochastic Lagrangian setting (and in particular to the Navier-Stokes equation) can be found in [41].

For the Euler equation, Brenier looked for probability measures $P$ on the path space $C([0, T] ; \mathcal{O})$ which minimize the energy functional

$$
\frac{1}{2} \int_{C([0, T] ; \mathcal{O})}\left[\int_{0}^{T}|\dot{\gamma}(t)|^{2} d t\right] d P(\gamma)
$$

with the incompressibility constraints $\left(e_{t}\right)_{*} P=d m(x)$, where $e_{t}: \gamma \rightarrow \gamma(t)$ is the evaluation map. A solution of such problem gives rise to a weaker type of Lagrangian flows for the Euler equation. Indeed one can define a probability measure $\mu$ on $[0, T] \times \mathcal{O}^{2}$ by

$$
\int f(t, x, v) d \mu=\frac{1}{T} \int_{C([0, T] ; \mathcal{O})} f\left(t, \gamma(t), \gamma^{\prime}(t)\right) d P(\gamma) d t
$$

and $\mu$ solves the Euler equation in a weak sense, namely

$$
\int\left[v \cdot w^{\prime}(x) \alpha^{\prime}(t)+v \cdot(\nabla w(x) \cdot v) \alpha(t)\right] d \mu(t, x, v)=0
$$

holding for all smooth test functions $\alpha(t)$ and every smooth divergence free vector field $v$. These kind of weak solutions of partial differential equations is known as solutions in the sense of Di Perna and Majda.

In the viscous case, we consider $\mathcal{O}$-valued semimartingales $g_{t}$ of the form $d g_{t}=\sqrt{2 v} d W_{t}+u_{t} d t$ (remark that the drift can be random) and their corresponding laws $\mathbb{P}^{g}$ on the path space $C([0, T] ; \mathcal{O})$ : for every cylindrical functional $F$,

$$
\int_{C([0, T], \mathcal{O})} F\left(\gamma\left(t_{1}\right), \ldots, \gamma\left(t_{n}\right)\right) d \mathbb{P}^{g}(\gamma)=\int_{\mathcal{O}}\left[\int_{C([0, T], \mathcal{O})} F\left(g_{t_{1}}(x), \ldots, g_{t_{n}}(x)\right) d \mathbb{P}_{x}^{g}\right] d m(x)
$$

where $\mathbb{P}^{g}=\mathbb{P}_{x}^{g} \otimes d m(x)$. Under $\mathbb{P}_{x}^{g}$, the semimartingale $g_{t}$ starts from $x$.

We say that the semimartingale $g_{t}$ is incompressible if, for each $t>0$,

$$
E_{\mathbb{P} g}\left[f\left(g_{t}\right)\right]=\int_{\mathcal{O}} f(x) d x, \quad \text { for all } f \in C(\mathcal{O})
$$

and we define the energy functional of a semimartingale $g$ by the formula

$$
\frac{1}{2} \int_{\mathcal{O}} E_{\mathbb{P}_{x}^{g}}\left(\int_{0}^{T}\left|D_{t} g(x)\right|^{2} d t\right) d m(x)=\frac{1}{2} \int_{\mathcal{O}} E_{\mathbb{P}_{x}^{g}}\left(\int_{0}^{T}\left|u_{t}\left(g_{t}(x)\right)\right|^{2} d t\right) d m(x)
$$

Considering suitably admissible variations we concluded in [41] that the semimartingales which are critical points of the energy functional above solve the Navier-Stokes equation in the following weak form:

$$
\int_{\mathcal{O}} \int_{0}^{T}<u_{t}, \alpha^{\prime}(t) w+\alpha(t) \nabla w \cdot u_{t}-v \alpha(t) \Delta w>d t d m(x)=0
$$

for all smooth functions of time $\alpha$ and all smooth vector fields $w$ such that $\operatorname{div}(w)=0$. Moreover we have showed that, in the case where $\mathcal{O}$ is the torus (corresponding to periodic boundary conditions) and under certain additional assumptions, classical solutions of the Navier-Stokes are minimizers of the energy action functional. 


\section{Stability Properties}

In finite dimensions it is well known that the behavior of geodesics can be expressed in terms of the curvature of the underlying manifold via the Jacobi equation.

Arnold's approach to the Euler equation allowed to show, in many cases, that the curvature of the spaces of diffeomorphisms is negative and therefore that the fluid trajectories are unstable (or "chaotic"), i.e., their distance, starting from different initial conditions, grows exponentially during time evolution (c.f [16]).

For viscous flows it is expected that particles become closer and closer after some possible initial stretching. For our model, at least in the case of the two dimensional torus, we could show ([23] and also [42]) that sensitivity with respect to initial conditions of the trajectories is enhanced by their stochasticity. The behavior will depend of the choice of diffusion coefficients that we consider in the Lagrangian paths or, in other words, on which scales and with what strength the motion is excited.

We have considered on the two-dimensional flat torus $\mathbb{T}=(\mathbb{R} / 2 \pi \mathbb{Z})^{2}$ a Brownian motion of the form

$$
d B_{t}=\sum_{k \in \mathbb{Z}^{2}}\left(k_{2},-k_{1}\right) \sqrt{v} \lambda_{k} A_{k} d W_{t}^{k}
$$

where $A_{2 k}(x)=\cos (2 k \cdot x), A_{2 k+1}(x)=\sin ((2 k+1) \cdot x), k \in \mathbb{Z}^{2}, x \in \mathbb{T}$. The coefficients $\lambda_{k}$ have to be such that the process $B$ is well defined, which is achieved by assuming a certain decay at infinity of those coefficients (see [22]). The Lagrangian stochastic processes associated to the Navier-Stokes equation will be defined as in Equation (7), with $W_{t}$ replaced by $B_{t}$.

Consider the following distance between trajectories:

$$
\rho^{2}(g, \tilde{g})=\int_{\mathbb{T}}|g(x)-\tilde{g}(x)|^{2} d m(x) .
$$

By using Itô calculus we have deduced, in particular, that under the assumption that the Navier-Stokes solution $u$ satisfies $\nabla u(t, x) \leq c_{1} e^{-c_{2} t}$, we have an estimate for the distance between trajectories of the form

$$
\rho_{t} \geq \rho_{s} \exp \left(Z_{t}+c_{3} t-\frac{c_{1}}{c_{2}}\left(1-e^{-c_{2} t}\right)\right.
$$

for $s \leq t$, where $Z$ is a 1-dimensional Brownian motion, $c_{3}$ another constant which depends on the coefficients $\lambda$.

The assumption on the gradient of $u$ implies that the velocity decays to zero at exponential rate. On the contrary the stochastic Lagrangian flows, describing the position of the fluid, get apart exponentially, at least for short times. Moreover, by the explicit expression of the constant $c_{3}$ ([23]), we observe that the stochastic Lagrangian trajectories for a fluid with a given viscosity constant tend to get apart faster when the higher Fourier modes (and therefore the smaller length scales) are randomly excited.

We can also show how the rotation of two particles, when their distance is small, becomes more and more irregular as time evolves, with explicit formulae in the torus case.

\section{A Stochastic Navier-Stokes Equation}

When we consider random forces, Navier-Stokes becomes a stochastic partial differential equation. In this paragraph we formulate some of these type of equations by a variational principle, where the action functional is suitably perturbed. 
We define the action functional, which is now a random variable (in particular we remove the expectation) with some extra stochastic terms in the Lagrangian, as

$$
\begin{aligned}
S_{\omega}(g, p) & =\frac{1}{2} \int_{0}^{T} \int\left|D_{t} g_{t}(x)\right|^{2} d t d m(x)+\int_{0}^{T} \int p\left(t, g_{t}(x)\right)\left(\operatorname{det} \nabla g_{t}(x)-1\right) d t d m(x) \\
& +\int_{0}^{T} \int D_{t} g_{t}(x) d M_{t}^{g}(x) d m(x)-\sqrt{2 v} \int_{0}^{T} \int D_{t} g_{t}(x) d W_{t} d m(x)
\end{aligned}
$$

where $M^{g}$ denotes the martingale part of the diffusion process $g$. We use the same variations as in Section 4.2. The variation of the two extra terms gives

$$
\int_{0}^{T} \int\left[\left(h\left(t, g_{t}\right) \cdot \sqrt{2 v} d W_{t}\right)+\left(D_{t} g_{t} \cdot\left(\nabla h\left(t, g_{t}\right) \cdot d W_{t}\right)\right)-\left(h\left(t, g_{t}\right) \cdot \sqrt{2 v} d W_{t}\right)\right] d m(x)
$$

that reduces to

$$
\begin{gathered}
\int_{0}^{T} \int v\left(t, g_{t}(x) \cdot\left(\nabla h\left(t, g_{t}(x)\right) \cdot d W_{t}\right) d x=\int_{0}^{T} v(t, x) \cdot\left(\nabla h(t, x) \cdot d W_{t}\right)\right. \\
=-\int_{0}^{T}\left((\nabla v(t, x) \cdot h(t, x)) \cdot d W_{t}\right)
\end{gathered}
$$

equality which holds for all $h, P$-almost surely.

Hence we obtain the following (c.f $[25,26])$,

Theorem 3. A diffusion $g_{t}$ of Equation (8) and a function $p$ are critical for the action functional $S_{\omega}$ if and only if the drift $u(t, \cdot)$ of $g_{t}$ satisfies the stochastic Navier-Stokes equation

$$
d_{t} u+(u . \nabla) u d t=\sqrt{2 v} \nabla u d W_{t}+v \Delta u d t-\nabla p d t, \quad \operatorname{div} u(t, \cdot)=0, \quad \nabla u \cdot n=0 \text { in } \partial \mathcal{O}
$$

with $t \in[0, T]$.

The stochastic Navier-Stokes equation written above can also be regarded as a stochastic perturbation of Euler equation in the sense that, replacing Itô differentials by Stratonovich ones, Equation (23) is equivalent to

$$
d_{t} u+(u . \nabla) u d t=\sqrt{2 v} \nabla u \circ d W_{t}-\nabla p d t, \quad \operatorname{div} u(t, \cdot)=0, \quad \nabla u . n=0 \text { in } \partial \mathcal{O}
$$

with $t \in[0, T]$ and where od stands for Stratonovich differential.

We remark that much more general noises can be considered and that we have only chosen a simple Brownian motion for simplicity. On the other hand the fact that we obtain a transport type noise is intrinsically related to our model.

There are many references for stochastic partial differential equations which are perturbations of Euler or Navier-Stokes. One can find in the recent work [5] a model where, as in our case, the noise is multiplicative.

In a general Lie group framework a variational approach to stochastic partial differential equations was developed in [25].

\section{Other Equations, Methods and Discussion}

The stochastic approach to Navier-Stokes described in this review can, in principle, be applied to describe any second order perturbation of a conservative first-order ordinary differential equation, when the latter is formulated in a suitable geometric way, as the Euler equation is formulated via a 
geodesic equation. This is the case, for example, for the Camassa-Holm equation, the Hunter-Saxton equation, the average Euler equation and the equations governing the motion of rigid bodies.

The viscous Camassa-Holm equation was studied in detail in [43]. It corresponds to replacing the $L^{2}(d m)$ metric, the energy in the Lagrangian, by the Sobolev $H^{1}$ metric and this equation reads

$$
\frac{\partial}{\partial t} v+(u \cdot \nabla) v=v \Delta v+\sum_{j} \nabla u^{j} \cdot \Delta u^{j}-\nabla p, \quad \operatorname{div} u=0
$$

where $v=u-\Delta u$.

Compressible Navier-Stokes or viscous Magnetohydrodynamical equations, on the other hand, have to be coupled with tracers (advected quantities) that need to be modeled by extra variables. Mathematically one introduces, together with the Lie group for the Lagrangian flows, a semidirect product vectorial structure (c.f [44]). We refer to [25] for a study of these various equations where we use stochastic methods, and stress again the fact that we can derive such equations by means of variational principles without any reference to thermodynamic considerations.

An extension of Arnold's geometric framework, describing compressible fluid dynamics (and other systems) with Newton's equations on a space of probability densities, can be found in the recent paper [45]. It reveals interesting connections with optimal transport and information theory, in particular.

The construction of solutions as critical points of the stochastic action functionals does not easily follow from the direct methods of the calculus of variations. We have instead indicated an indirect approach based on forward-backward stochastic differential equations, but even there we encountered many technical difficulties. Other possibilities are the use of entropy methods, as described in Section 5.2, or the relaxation of the notion of solution (c.f Section 5.3).

For conservative dynamical systems, it is well known that one can take advantage of the symmetries of such systems to reduce the complexity of the equations. Symmetries also play an important role in the implementation of numerical algorithms designed to investigate the equations in question. The natural objects to be introduced in order to replace constants of motion are martingales, since a martingale $M$, by definition, is a quantity whose generalized derivative vanishes $\left(D_{t} M=0\right)$. One can find a Noether theorem for a certain class of diffusion processes in [46]. We refer to [47] for a brief discussion on symmetries in our context.

Finally, let us point out that numerical methods tied up with our probabilistic approach still have to be developed.

Funding: This research was partly funded by FCT (Fundação para a Ciência e Tecnologia, Portugal), grant "Schrödinger's problem and Optimal Transport: a multidisciplinary perspective", with reference PTDC/MAT-STA/28812/2017.

Conflicts of Interest: The author declare no conflict of interest.

\section{Appendix A. Some Basic Notions of Stochastic Calculus}

We start by recalling the definition of a ( $\mathbb{R}$-valued) Brownian motion $W_{t}, t \in[0, T]$. This is a continuous stochastic process (namely, a function of time and of a random parameter in some standard probability space $(\Omega, \mathcal{B}, P))$ that verifies the following properties:

(i) $\quad W_{0}=x$;

(ii) $\quad W_{t}$ has independent increments;

(iii) For $s<t, W_{t}-W_{s}$ has a normal distribution $\mathcal{N}(0, t-s)$.

A $\mathbb{R}^{d}$ Brownian motion corresponds to a collection of independent and identically distributed copies of one dimensional Brownian motions. A Brownian motion is also called a Wiener process.

We generally omit the random variable in the notation when we write a stochastic process. 
Suppose that the probability space is endowed with a filtration, namely an increasing family $\mathcal{P}_{t}$ of sub $\sigma$-algebras of $\mathcal{B}$. Typically each $\mathcal{P}_{t}$ represents the events that occur before a time $t$. A stochastic process $M_{t}$ is a ( $\mathbb{R}$-valued) martingale with respect to $\mathcal{P}_{t}$ if

(i) $\quad M_{t}$ is $\mathcal{P}_{t}$-measurable for all $t$ (we also say that $M_{t}$ is adapted to $\mathcal{P}_{t}$ );

(ii) $\quad E\left|M_{t}\right|<+\infty$;

(iii) For $s<t, E_{s}\left(M_{t}\right)=M_{s}$. Here $E$ and $E_{t}$ denote respectively expectation and conditional expectation with respect to $\mathcal{P}_{t}$. Multidimensional martingales are defined analogously.

A real-valued Brownian motion can also be characterized as a martingale with continuous sample paths such that, for all $t, W_{t}^{2}-t$ is also a martingale.

When a martingale is (a.s.) continuous in time and satisfies the assumption $E\left|M_{t}\right|^{2}<+\infty$, we define its quadratic variation as the limit, in probability, of the sums

$$
<M, M>_{t}=\sum_{t_{i}, t_{i+1}}\left(M_{t_{i+1}}-M_{t_{i}}\right)^{2}
$$

when the mesh of the partition $\left\{t_{i}\right\}$ goes to zero. For a Brownian motion defined in $[0, T]$ and starting from zero, this limit is equal to $T$. One defines the covariation between two martingales $M_{t}$ and $N_{t}$ as the limit of the sums

$$
<M, N>_{t}=\sum_{t_{i}, t_{i+1}}\left(M_{t_{i+1}}-M_{t_{i}}\right)\left(N_{t_{i+1}}-N_{t_{i}}\right) .
$$

A stochastic process $X_{t}$ is a semimartingale if, for every $t$ it can be decomposed into a sum

$$
X_{t}=M_{t}+A_{t}
$$

of a martingale $M_{t}$ and a process of bounded variation (or almost-surely differentiable) $A_{t}$, with $A_{0}=0$ a.s. The martingale part describes a diffusion, the bounded variation part an evolution which is "similar" to a deterministic one. The quadratic variation of a semimartingale is, by definition, equal to the quadratic variation of its martingale part.

Martingales and, in particular, Brownian motion, are (almost surely) never differentiable in time; therefore one cannot integrate with respect to them with a Lebesgue-Stieltjes kind of integration. Itô introduced a theory of stochastic integration, now named Itô's stochastic calculus. The Itô's integral is defined by the following limit

$$
\int_{0}^{t} X(s) d W(s)=\lim \sum_{t_{i}, t_{i+1}} X\left(t_{i}\right)\left(W_{t_{i+1}}-W_{t_{i}}\right),
$$

the limit being taken in probability and when we consider partitions of the time interval $[0, t]$ with mesh converging to zero. Itô's integral is well defined when $X_{t}$ is a semimartingale such that $E\left|X_{t}\right|^{2} d t<+\infty$.

Notice that the values of the integrand $X_{t}$ are taken on the left point of the intervals $\left[t_{i}, t_{i+1}\right]$. Unlike in usual Lebesgue-Stieltjes integration, considering its values in any other point of these intervals leads to completely different results. Another common and interesting way to define a stochastic integral is the so-called Stratonovich integral:

$$
\int X(s) \circ d W(s)=\lim \frac{1}{2} \sum_{t_{i}, t_{i+1}}\left(X\left(t_{i}\right)+X_{t_{i+1}}\right)\left(W_{t_{i+1}}-W_{t_{i}}\right) .
$$

Each type of integrals present its own advantages. Although Stratonovich integral demands more regularity on the integrand in order to be well defined, it is a more intrinsic concept and more adapted to be extended to semimartingales with values in curved spaces, for example. Also, as we shall write below, the rules of differential calculus are analogous to the classical ones for these integrals (and 
quite different for the Itô integral), which makes Stratonovich somehow popular in applications or in Physics. Nevertheless it turns out that $\int_{0}^{t} X(s) d W(s)$ is a martingale, where $\int_{0}^{t} X(s) \circ d W(s)$ is not, in general. More important for us, any martingale is in fact a stochastic Itô integral and therefore any semimartingale can be decomposed into an Itô integral representing a diffusion, or a pure fluctuation, and a bounded variation part that represents the mean dynamical content of the process. So even though, when both integrals are defined, they are related by the following formula,

$$
\int_{0}^{t} X(s) \circ d W(s)=\int_{0}^{t} X(s) d W(s)+\frac{1}{2} \int_{0}^{t} d<X, W>_{s},
$$

dynamics is better identified using Itô integration.

Rules of differentiation are given by the so-called Itô's formula. If $f$ is a regular function and $X_{t}$ a $\left(\mathbb{R}^{d}\right.$ valued) semimartingale,

$$
f\left(X_{t}\right)=f\left(X_{0}\right)+\sum_{i=1}^{d} \int_{0}^{t} \frac{\partial}{\partial x_{i}}\left(X_{s}\right) d X^{i}(s)+\frac{1}{2} \sum_{i, j=1}^{d} \int_{0}^{t} \frac{\partial^{2}}{\partial x_{i} \partial x_{j}} f\left(X_{s}\right) d<X^{i}, X^{j}>_{s}
$$

or, in differential form,

$$
d f\left(X_{t}\right)=\sum_{i=1}^{d} \frac{\partial}{\partial x_{i}}\left(X_{t}\right) d X^{i}(t)+\frac{1}{2} \sum_{i, j=1}^{d} \frac{\partial^{2}}{\partial x_{i} \partial x_{j}} f\left(X_{t}\right) d<X^{i}, X^{j}>_{t}
$$

For Stratonovich integrals we have, as in usual differential calculus,

$$
d f\left(X_{t}\right)=\sum_{i=1}^{d} \frac{\partial}{\partial x_{i}}\left(X_{t}\right) \circ d X^{i}(t)
$$

If $f$ is time dependent one has to add the term $\frac{\partial f}{\partial t}\left(t, X_{t}\right) d t$ in the formulae.

Recall that, in the case of independent Brownian motions $W_{t}^{i}$, and writing $\operatorname{id}(t)=t$, we have

$$
d<W^{i}, W^{j}>_{t}=\delta_{i j} d t, \quad d<W^{i}, \text { id }>_{t}=0 \forall i .
$$

Stochastic differential equations generalize ordinary differential ones. Given $\sigma$ with values in matrices of type $d \times r$, a vector field (possibly time-dependent) $u$ and an initial random variable $X_{0}$, these equations take the form

$$
d X_{t}=\sigma\left(X_{t}\right) \cdot d W(t)+u\left(t, X_{t}\right) d t .
$$

Using Itô's formula we deduce that, if $X_{t}$ is a solution of such an equation and $X_{0}=x$, we have, for a smooth function $f$,

$$
\lim _{t \rightarrow 0} \frac{1}{t} E_{x}\left(f\left(X_{t}\right)-f(0, x)\right)=\mathcal{L} f(x),
$$

where $\mathcal{L}$ is the second order linear operator

$$
\mathcal{L} f(x)=\frac{1}{2} \sum_{i, j}\left(\sigma \sigma^{T}\right)_{i}^{j} \frac{\partial^{2} f}{\partial x_{i} \partial x_{j}}+(u . \nabla f) .
$$

The operator $\mathcal{L}$ is called the generator of the process $X_{t}$.

Solutions of stochastic differential equations are particular cases of semimartingales. They are defined globally in time when the coefficients $\sigma$ and $u$ are smooth and bounded. Many works, even very recent ones, have been devoted to extend existence of solutions for less regular coefficients, in particular of Sobolev type. 
When considering diffusion processes on domains rather than in all the Euclidean space, and subject to boundary conditions, we need to use the notion of local time. The local time of a Brownian motion is a family of (a.s.) continuous non negative random variables $\ell(t, x)$ such that, for any set $A$ and $t>0$ we have

$$
\int_{0}^{t} 1_{A}\left(W_{s}\right) d s=2 \int_{A} \ell(t, x) d m(x) .
$$

It may also be regarded as the limit

$$
\ell(t, x)=\lim _{\mathcal{E} \rightarrow 0} \frac{1}{4 \varepsilon} \int_{0}^{t} 1_{] x-\varepsilon, x+\varepsilon[}\left(W_{s}\right) d s .
$$

Local time corresponds to the amount of time spent by a Brownian path in a neighborhood of a point $x \in \mathbb{R}$. This concept was introduced by Paul Lévy in 1948 (see, for example [48]). The concept extends naturally to the multidimensional case as well as to semimartingales $X_{t}$. We have,

$$
\int_{0}^{t} f\left(X_{t}\right) d<X, X>_{t}=2 \int_{-\infty}^{+\infty} f(x) \ell(t, x) d m(x)
$$

for every regular function $f$, where $\ell(t, x)=\int_{0}^{t} 1_{\partial \mathcal{O}}\left(X_{s}(x)\right) d \ell(s)$. Then we can consider stochastic differential equations in domains $\mathcal{O} \subset \mathbb{R}^{d}$, of the form

$$
d X_{t}=\sigma\left(X_{t}\right) \cdot d W(t)+u\left(t, X_{t}\right) d t+n\left(X_{t}\right) d \ell(t), \quad X_{0}=x,
$$

where $n$ denotes the unitary vector normal to the boundary. These stochastic processes are reflected at the boundary and, in terms of partial differential equations (their generators), they correspond to considering Neumann boundary conditions.

Finally we consider the more recent notion of forward-backward stochastic differential equation (cf., for example [49]).

For given (smooth) coefficients $\sigma, u, v$, initial condition $X_{0}$ and final condition $h$, one looks for semimartingales $X_{t}, Y_{t}, t \in[0, T]$ which are solutions of the following system of stochastic differential equations (written here in integral form),

$$
\left\{\begin{array}{l}
X_{t}=X_{0}+\int_{0}^{t} \sigma\left(X_{s}\right) \cdot d W_{s}+\int_{0}^{t} u\left(s, X_{s}\right) d s \\
Y_{t}=h\left(X_{T}\right)-\int_{t}^{T} Z_{s} \cdot d W_{s}+\int_{t}^{T} v\left(s, X_{s}, Y_{s}, Z_{s}\right) d s
\end{array}\right.
$$

with $E \int_{0}^{T}\left[\left|X_{t}\right|^{2}+\left|Y_{t}\right|^{2}+\left|Z_{t}\right|^{2}\right] d t<\infty$. There are two remarkable features of such systems: one is that, in spite of a condition given at a final time $(h)$, these solutions turn out, in fact, to be adapted to the past filtration. Another one is that, even if a priori we have three unknowns $X, Y$ and $Z$, the last one will be in fact equal to $Z_{t}=\nabla u\left(X_{t}\right)$. These kind of systems are natural generalizations of second order ordinary differential equations to the stochastic setting.

\section{References}

1. Marchioro, C.; Pulvirenti, M. Vortex methods in two-dimensional fluid mechanics. In Lecture Notes in Physics; Springer: Berlin Germany, 1984

2. Vishik, M.I.; Komechi, A.I.; Fursikov, A.I. Some mathematical problems of statistical hydrodynamics. Russ. Math. Surv. 1979, 34, 149-234. [CrossRef]

3. Gawedzki, K. Soluble models of turbulent transport. In Non-Equilibrium Statistical Mechanics and Turbulence; Nazarenko, S., Zaboronski, O., Eds.; Cambridge Uniersity Press: Cambridge, UK, 2008; pp. 47-107.

4. Palmer, T.N.; Williams, P.D. Introduction. Stochastic physics and climate modelling. Philos. Trans. R. Soc. A 2008, 366, 2421-2427. [CrossRef] [PubMed]

5. Crisan, D.; Flandoli, F.; Holm, D.D. Solution properties of a 3D stochastic Euler Fluid equation. J. Nonlin. Sci. 2019, 29, 813-870. [CrossRef] 
6. Bensoussan, A.; Teman, R. Équations stochastiques du type Navier-Stokes. J. Funct. Anal. 1973, 13, $195-222$. [CrossRef]

7. Pope, S.B. On the relationship between stochastic Lagrangian models of turbulence and second-moment closures. Phys. Fluids 1994, 6, 973-985. [CrossRef]

8. Holm, D.D. Variational principles for stochastic fluid dynamics. Proc. R. Soc. A 2015, 471, 20140963. [CrossRef]

9. Busnello, B. A probabilistic approach to the two-dimensional Navier-Stokes equation. Ann. Probab. 1999, 27, 1750-1780. [CrossRef]

10. le Jan, Y.; Sznitman, A.S. Stochastic cascades and the 3-dimensional Navier-Stokes equations. Probab. Theory Relat. Fields 1997, 109, 343-366. [CrossRef]

11. Constantin, P.; Iyer, G. A stochastic Lagrangian representation of the three-dimensional incompressible Navier-Stokes equation. Commun. Pure Appl. Math. 2008, 61, 330-345. [CrossRef]

12. Nakagomi, T.; Yasue, K.; Zambrini, J.-C. Stochastic variational derivations of the Navier-Stokes equation. Lett. Math. Phys. 1981, 160, 337-365. [CrossRef]

13. Yasue, K. A variational principle for the Navier-Stokes equation. J. Funct. Anal. 1983, 51, 133-141. [CrossRef]

14. Arnold, V.I. Sur la géométrie différentielle des groupes de Lie de dimension infinie et ses applications à l'hydrodynamique des fluides parfaits. Ann. Inst. Fourier 1966, 16, 316-361. [CrossRef]

15. Ebin, D.G.; Marsden, J.E. Groups of diffeomorphisms and the motion of an incompressible fluid. Ann. Math. 1970, 17, 102-163. [CrossRef]

16. Arnold, V.I.; Khesin, B.A. Topological Methods in Hydrodynamics; Springer: Berlin, Germany, 1998.

17. Eyink, K.L. Stochastic line motion and stochastic flux conservation for nonideal hydromagnetic models. J. Math. Phys. 2009, 50, 083102. [CrossRef]

18. Koide, T.; Kodama, T. Navier-Stokes, Gross-Pitaevskii and generalized diffusion equations using the stochastic variational method. J. Math. A 2012, 45, 255204. [CrossRef]

19. Ikeda, N.; Watanabe, S. Stochastic Differential Equations and Diffusion Processes; Springer: Berlin, Germany, 1981.

20. Marsden, J.E.; Ratiu, T.S. Introduction to Mechanics and Symmetry: A Basic Exposition of Classical Mechanical Systems. In Texts in Applied Mathematics; Springer: Berlin, Germany, 1999; Volume 17.

21. Nelson, E. Dynamical Theories of Brownian Motion; Princeton University Press: Princeton, NJ, USA, 1967.

22. Cipriano, F.; Cruzeiro, A.B. Navier-Stokes equation and diffusions on the group of homeomorphisms of the torus. Commun. Math. Phys. 2007, 275, 255-269. [CrossRef]

23. Arnaudon, M.; Cruzeiro, A.B. Lagrangian Navier-Stokes diffusions on manifolds: variational principle and stability. Bull. Sci. Math. 2012, 136, 857-881. [CrossRef]

24. Arnaudon, M.; Chen, X.; Cruzeiro, A.B. Stochastic Euler-Poincaré reduction. J. Math. Phys. 2014, 55, 081507. [CrossRef]

25. Chen, X.; Cruzeiro, A.B.; Ratiu, T.S. Stochastic variational principles for dissipative equations with advected quantities. arXiv 2018, arXiv:1506.05024.

26. Cruzeiro, A.B. Navier-Stokes and stochastic Navier-Stokes equations via Lagrange multipliers. J. Geom. Mech. 2019, 11, 553-560. [CrossRef]

27. Latas, M. On the Derivation of the Navier-Stokes Equations from a Nondeterministic Variational Principle. Master's Thesis, University of Lisbon, Lisbon, Portugal, 2019.

28. Cruzeiro, A.B.; Shamarova, E. Navier-Stokes equations and forward-backward SDEs on the group of diffeomorphisms of a torus. Stoch. Proc. Their Appl. 2009, 119, 4034-4060. [CrossRef]

29. Cruzeiro, A.B.; Qian, Z. Backward stochastic diferential equations associated with the vorticity equations. J. Funct. Anal. 2014, 267, 660-677. [CrossRef]

30. Chen, X.; Cruzeiro, A.B.; Qian, Z. Navier-Stokes equation and forward-backward stochastic differential system in the Besov spaces. arXiv 2013, arXiv:1305.0647.

31. Douglas, J.; Ma, J.; Protter, P. Numerical methods for forward-backward stochastic differential equations. Ann. Appl. Prob. 1996, 6, 940-968. [CrossRef]

32. Lejay, A.; González, H.M. A Forward-Backward Probabilistic Algorithm for the Incompressible Navier-Stokes Equations. 2019. Available online: https:/ / hal.inria.fr/hal-02377108 (accessed on 26 February 2020).

33. Chen, X.; Cruzeiro, A.B. Stochastic geodesics and forward-backward stochastic differential equations on Lie groups. Discrete Contin. Dyn. Syst. 2013, 115-121. [CrossRef] 
34. Arnaudon, M.; Cruzeiro, A.B.; Léonard, C.; Zambrini, J.-C. An entropic interpolation problem for incompressible viscid fluids. Ann. Inst. H. Poincaré, 2019.

35. Schrödinger, E. Sur la théorie relativiste de l'electron et l'interprétation de la mécanique quantique. Ann. Inst. H. Poincaré 1932, 2, 269-310.

36. Zambrini, J.-C. Stochastic mechanics according to E. Scrödinger. Phys. Rev. A 1986, 33, 1532-1548. [CrossRef]

37. Léonard, C. A survey of the Scrödinger problem and some of its connections with optimal transport. Discrete Contin. Dyn. Syst. 2014, 34, 1533-1574. [CrossRef]

38. Villani, V. Optimal Transport. Old and New; Springer: Wissen, Germany, 2009.

39. Brenier, Y. A homogenized model for vortex sheets. Arch. Ration. Mech. Anal. 1997, 138, 319-353. [CrossRef]

40. Brenier, Y. The least action principle and the related concept of generalized flows for incompressible perfect fluids. J. Am. Math. Soc. 1989, 2, 225-255. [CrossRef]

41. Arnaudon, M.; Cruzeiro, A.B.; Fang, S. Generalized stochastic Lagrangian paths for the Navier-Stokes equation. Ann. Sci. Norm. Super. Pisa Cl. Sci. 2018, 18, 1033-1060.

42. Arnaudon, M.; Cruzeiro, A.B.; Galamba, N. Lagrangian Navier-Stokes flows: a stochastic model. J. Phys. A Math. Theory 2011, 44, 175501. [CrossRef]

43. Cruzeiro, A.B.; Liu, G. A stochastic variational approach to the viscous Camassa-Holm and Leray-alpha equations. Stoch. Proc. Their Appl. 2017, 127, 1-19. [CrossRef]

44. Holm, D.D.; Marsden, J.E.; Ratiu, T.S. The Euler-Poincaré equations and semidirect products with applications to continuum mechanics. Adv. Math. 1998, 137, 1-81. [CrossRef]

45. Khesin, B.; Misiolek, G.; Modin, K. Geometric hydrodynamics via Mandelung transform. PNAS 2018, 115, 6165-6170. [CrossRef]

46. Thieullen, M.; Zambrini, J.-C. Probability and quantum symmetries. I. The theorem of Noether in Schrödinger's Euclidean quantum mechanics. Ann. Inst. H. Poincaré Phys. Théorique 1997, 67, 297-338.

47. Cruzeiro, A.B.; Lassalle, R. Symmetries and martingales in a stochastic model for the Navier-Stokes equation. In From Particle Systems to PDEs III; Springer: Berlin, Germany, 2016; Volume 162, pp. 185-194.

48. Pilipenko, A. An Introduction to Stochastic Differential Equations with Reflection; Postdam University Press: Postdam, Germany, 2014.

49. Ma, J.; Yong, J. Forward-backward stochastic differential equations and their applications. In Lecture Notes in Math; Springer: Berlin, Germany, 2007.

(C) 2020 by the authors. Licensee MDPI, Basel, Switzerland. This article is an open access article distributed under the terms and conditions of the Creative Commons Attribution (CC BY) license (http://creativecommons.org/licenses/by/4.0/). 